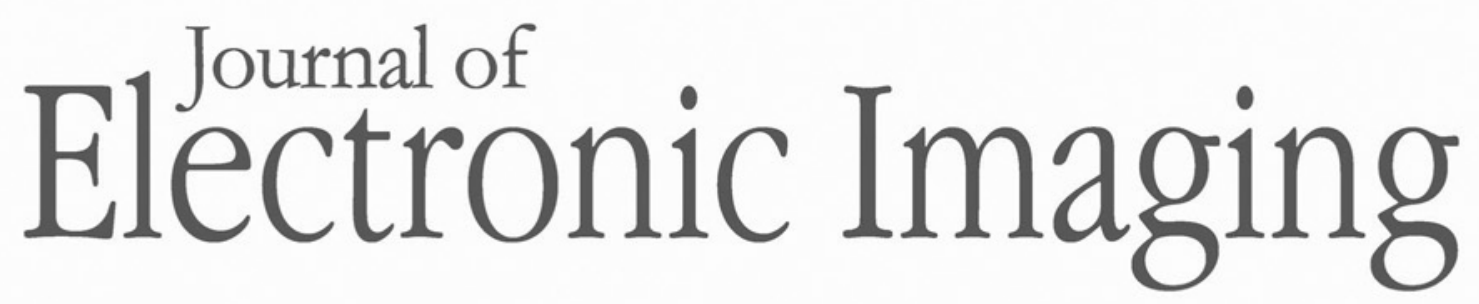

JElectroniclmaging.org

\title{
JEI 2014 List of Reviewers
}


The Journal of Electronic Imaging would like to sincerely thank the following individuals who served as reviewers in 2014 . The success of our publication hinges on the voluntary contributions of time and energy put forth by these professionals.

\begin{tabular}{|c|c|c|}
\hline Aabdelah Aarab & Jose Boluda & Ngai-man Cheung \\
\hline Ayman Abaza & Nicolas Bonnier & Teodora Chitiboi \\
\hline Almas Abbasi & Ajit Bopardikar & John Chiverton \\
\hline Jaafar Abdullah & Prabin Kumar Bora & Nam Ik Cho \\
\hline Mehrdad Abolbashari & Giacomo Boracchi & Kiho Choi \\
\hline Sergey Abramov & Glenn Boreman & Yoonsuk Choi \\
\hline Begoa Acha & Frederic Borne & Roya Choupani \\
\hline Tinku Acharya & Frank Bossen & Ananda Chowdhury \\
\hline Andy Adler & Mohamed-Salim Bouhlel & Julianne Chung \\
\hline Jianhua Adu & Don Bouwhuis & Ryan Close \\
\hline Parthipan Aiva & Abdesselam Bouzerdoum & Rami Cohen \\
\hline Igor Aizenberg & Salim Bouzerdoum & Miguel Colom \\
\hline Lale Akarun & Alan Bovik & Pedro Comesana Alfaro \\
\hline Zahid Akhtar & John P. Boyd & Diane Cook \\
\hline Mahmood Al-Khassaweneh & Brett Bradley & David Corcoran \\
\hline Hazem Al-Otum & Michael Brill & Ahmet Coskun \\
\hline Ahmed Al-Zubaidi & Anna Brook & Manuel Costa \\
\hline Nicolas Alvertos & Arcangelo Bruna & Vincent Courboulay \\
\hline Pierre Ambs & Adnan Bubalo & Chris Cox \\
\hline Irene Amerini & Katja Buehler & Timothy Crimmins \\
\hline Gideon Amir & Orhan Bulan & Gabriel Cristobal \\
\hline Le An & Aaron Burry & Vladimir Crnojevic \\
\hline Christos-Nikolaos Anagnostopoulos & Andrew Busch & Lorenzo Crocco \\
\hline Nantheera Anantrasirichai & Fernando Caballero & Eduardo Cuesta \\
\hline Gholamreza Anbarjafari & Xiaohao Cai & Sarada Dakua \\
\hline Mehran Andalibi & Gustavo Callico & Nils Damaschke \\
\hline Ludovic Angot & James Cambier & Niranjan Damera-Venkata \\
\hline Arathi Arakala & Kenneth Camilleri & Lorina Dascal \\
\hline Gonzalo Arce & Gang Cao & Bryan Davidson \\
\hline Carlos Ardao & Guo Cao & Abe Davis \\
\hline Paul Ardis & Alessandro Capra & Juan de Dios \\
\hline Henry Arguello & Joao Carreira & Alessandro De Mauro \\
\hline Andreas Arnold & Bruno Carvalho & Samuel de Sousa \\
\hline Muhammad Asad & Benjamin Castaneda & Christian Debes \\
\hline Hasan Ates & Mehmet Celenk & Walter Delashmit \\
\hline Cheung Auyeung & Turgay Celik & Alexander Denecke \\
\hline Aykut Avci & Mufit Cetin & Sandor Der \\
\hline Tayfun Aytac & Afef Chammem & Soojey Deshpande \\
\hline Enrico Baccaglini & Vincent $\mathrm{H}$. Chan & Agnes Desolneux \\
\hline Zachi Baharav & Damon Chandler & Frederic Devernay \\
\hline Hongliang Bai & Vinod Chandran & Michael DeWeert \\
\hline Donald Bailey & Kartheek Chandu & Arindam Dey \\
\hline Steve Bako & Chih-Hsiang Chang & Martin Dietze \\
\hline Jim Bankoski & Ming Chang & Mariella Dimiccoli \\
\hline Paul Bao & Pao-Chi Chang & Kamil Dimililer \\
\hline Leah Bar & Sumohana Channappayya & C.N. Dinakardas \\
\hline James Barabas & Vincent Chapdelaine-Couture & Giovanni Diraco \\
\hline Marcus Barkowsky & Avishek Chatterjee & Yakov Diskin \\
\hline Kenneth J. Barnard & Kunal Chaudhury & Ajay Divakaran \\
\hline Bernardino Barrientos & Chia-Yen Chen & Leila Djerou \\
\hline Atanu Basu & Hanfeng Chen & Julia Dobrosotskaya \\
\hline Sebastiano Battiato & Jie Chen & Evan Dong \\
\hline Federica Battisti & Lidong Chen & Ganggang Dong \\
\hline Sevinc Bayram & Wei Chen & Jing Dong \\
\hline Azeddine Beghdadi & Wen Chen & Weisheng Dong \\
\hline Ardhendu Behera & Wen Yuan Chen & Yiqiu Dong \\
\hline Amir Benzaoui & Wen-Jan Chen & Yongsheng Dong \\
\hline Kathrin Berkner & Xinjian Chen & Amer Draa \\
\hline Stefano Berretti & Ying Chen & Bo Du \\
\hline Nilanjana Bhattacharya & Yueting Chen & Jizhong Duan \\
\hline Mrinal Bhowmik & Zikuan Chen & Sylvain Durand \\
\hline Ning Bi & Beibei Cheng & Martin Ehler \\
\hline Li Bing & Erkang Cheng & Mohamed El hajji \\
\hline Philip Bingham & Fan-Chieh Cheng & Mireille El-Gheche \\
\hline A.A. Bini & Wu Cheng & Abderrahmane Elyousfi \\
\hline Soma Biswas & Ravindranath Cherukuri & Ulrich Engelke \\
\hline Henryk Blasinski & Aladine Chetouani & II Kyu Eom \\
\hline
\end{tabular}


Ozgur Ergul

Sezer Erkilinc

Georgios Evangelidis

Tim Faltemier

Wentao Fan

Chengyu Fang

Jiangxiong Fang

Jiunn-Tsair Fang

Ruogu Fang

Yuming Fang

Mylene Farias

Giovanni Farinella

Susan Farnand

Sina Farsiu

Ivar Farup

Eric Fauvet

Gui Feng

Guocan Feng

Steven Fernandes

Carlos Fernandez-Granda

Alicia Fernandez-Oliveras

A. Firouzian

Mani Fischer

Robert Fisher

Leonardo Florez Valencia

Antonio Foncubierta Rodriguez

Irene Fondon

Adria Fores Herranz

Alicia Fornes

Franco Frattolillo

Ichiro Fujinaga

Jacob Furst

Prasad Gabbur

Xiong Gang

Chenqiang Gao

Marie-Neige Garcia

Francesca Gasparini

Frans Gaykema

Xiaoyin $\mathrm{Ge}$

Marc Geese

Joe Geigel

Jason Geng

Gianluca Gennarelli

Zeno Geradts

Christan Germain

Izidor Gertner

Jerome Gilles

Andrew Gilman

Oliver Giudice

Georgi Gluhchev

Yildirim Gokhan

Geoffrey Goldman

Arash Golibagh Mahyari

Jay Gondek

Michael Gormish

Yves Goussard

Puneet Goyal

Maria Greco

Artyom Grigoryan

William Grosky

Lingjia Gu

Zhouye Gu

Mirko Guarnera

Jie Gui

M. Kemal Gullu

Gokhan Gultekin

Feng Guo

Guodong Guo

Jing-Ming Guo

Yulan Guo
Prudhvi Gurram

Mohammed Hachama

Ofer Hadar

Oren Haik

Tobias Haist

Raouf Hamzaoui

Tony X. Han

Philippe Hanhart

Miles Hansard

Pengwei Hao

M. Hassaballah

Soren Hauberg

Nicolas Hautiere

Chuangjiang $\mathrm{He}$

Huiguang $\mathrm{He}$

Xiaofei $\mathrm{He}$

Zhe He

Rodney Heckaman

Elsayed Hemayed

Keita Hirai

Manjunatha Hiremath

Ernest Hirsch

David M. Hoffman

S. Hojjatoleslami

Timothy Hospedales

Tobias Hossfeld

Rein Lien (Vincent) Hsu

Changbo $\mathrm{Hu}$

Keli Hu

Shuowen $\mathrm{Hu}$

Sudeng $\mathrm{Hu}$

Xinda Hu

Yu-Chen $\mathrm{Hu}$

Deng-Yuan Huang

Hui-Yu Huang

Jia-Bin Huang

Junzhou Huang

Shih-Chia Huang

Yong-Ren Huang

Ji Hui

Kwok-Wai Hung

Heikki Huttunen

Satoshi Ikehata

Tetsuri Inoue

Bogdan lonescu

Kashif lqbal

John Irvine

Akira Ishii

Paul Issartel

Robert Ives

Ioannis Ivrissimtzis

Euee Seon Jang

Zbigniew Jaroszewicz

Stuart Jefferies

Zhengping Ji

Hongjun Jia

Jie Jia

Jongjun Jia

Sun Jiande

Gangyi Jiang

Huaizu Jiang

Shaofeng Jiang

P. Jidesh

Guoxin Jin

Graham Johnson

Andrew Jones

Edward Jones

Graeme A. Jones

José Joskowicz

Yanglin Ju
Jae-Hyun Jung

Yong Ju Jung

Hideki Kakeya

Ajith Kamath

Hyun Soo Kang

Mohan Kankanhalli

Chi-Chou Kao

Damian Karwowski

Ergina Kavallieratou

Michal Kawulok

Masato Kazui

Hugh Kennedy

Saad Khan

Mohammed Lamine Kherfi

Byung-Gyu Kim

Changil Kim

Hui Yong Kim

Jaihie Kim

Joohwan Kim

Sungho Kim

Vladimir Kim

Hanseok Ko

Jonathan Kofman

Lisimachos P. Kondi

Adams W. K. Kong

Weiwei Kong

Hyung II Koo

Ivica Kopriva

Pavel Korshunov

Markus Koskela

Aikaterini Kotrotsou

Levente Kovacs

Samuel Kozaitis

Janusz Kozlowski

Iztok Kramberger

Alex Krizhevsky

Jiangtao Kuang

Toshiro Kubota

Dhananjay Kumar

Jayant Kumar

Naveen Kumar

Pradeep Kumar

Ravinder Kumar

Hideyasu Kuniba

R. Sanjeev Kunte

Holger Kunze

Do-Kyoung Kwon

Mikko Kyto

Demetrio Labate

Patricia Ladret

Ryan Lagerstrom

Khin Wee Lai

Zhi Hui Lai

Kenneth Lami

James Larimer

Olivier Le Meur

Epiphany Leavline

Pierre Lebreton

Byung-Uk Lee

Chan su Lee

Chul Lee

Chul Lee

Sangkeun Lee

Sangyoun Lee

Seok-Han Lee

Seungyong Lee

Suk-Ho Lee

Ido Leichter

Michael Leigsnering

Lasse Lensu 
Alessandro Leone José Lerma

Baoxin $\mathrm{Li}$

Changchun $\mathrm{Li}$

Chaorong $\mathrm{Li}$

Haoxiang $\mathrm{Li}$

Hongyu $\mathrm{Li}$

Jing Li

Jun-Bao Li

Leida Li

$\mathrm{Mu} \mathrm{Li}$

Peng Li

Pingshan $\mathrm{Li}$

Qiang Li

Sheng Li

Shutao Li

Wei Li

Xiaowei Li

Xin Li

Jie Liang

Chang Hong Lin

Chu-Fang Lin

Chunyu Lin

Yih-Chuan Lin

Baiyang Liu

Bin Liu

Chenguang Liu

Wanyu Liu

Weiliang Liu

Xianming Liu

Xiaobai Liu

Yanwei Liu

Ying Liu

Yipeng Liu

Zheng Liu

Zheng Liu

Zhengjun Liu

Juan Pedro Lopez

Huchuan Lu

Jiwen Lu

Shijian Lu

Xiusheng Lu

Yves Lucas

Vladimir Lukin

Guoqi Luo

Xiaoyan Luo

Andy Jinhua Ma

Jianwei Ma

$\mathrm{Li} \mathrm{Ma}$

Rui Ma

Siwei Ma

Tao Ma

Zhan Ma

Ludovic Macaire

Constantino Malagon

Luke Mander

Alamin Mansouri

Claire Mantel

Rafal Mantiuk

Thibault Marin

Diego Martinez Plasencia

Nor Ashidi Mat Isa

Nelson Max

Stephen Maybank

Mohammad Mayyas

Riccardo Mazzon

John J. McCann

Robert McFeely

Jeffrey McGough

John Mclntire
Stephen McNeill

Peter Meerwald

E. Menaka

Jorge Mendiola-Santibanez

Gaofeng Meng

Fabrice Meriaudeau

Giuseppe Messina

Jean Meunier

Xiaodong Miao

Simone Milani

Byungseok Min

Frank Mindrup

Xuan Mo

Bijan Mobasseri

Pranab Mohanty

Andrew Moore

Anush Moorthy

Yaileth Morales

Brendan Tran Morris

Daniel D. Morris

Tim Morris

Dibyendu Mukherjee

Karsten Muller

Alejandro Murua

Damien Muselet

Tanzeem Muzaffar

Soyeb Nagori

Binu Nair

Hammadi Nait-Charif

Manish Narwaria

Ambarish Natu

Rodrigo Nava

Mahdi Nezamabadi

Michael Ng

Lam Nguyen

Karl S. Ni

James Nightingale

Vincent Noblet

V. Nourrit

Paulo Nunes

Gote Nyman

Laszlo Nyul

Marek Ogiela

Stefan Ohrhallinger

Jeffrey Oliver

S. H. Ong

Maria Ortiz Segovia

Victor Ostromoukhov

Ming Ouhyoung

Suleyman Ozarslan

Carla Pagliari

Peshala Pahalawatta

Roman Palenychka

Dmitriy Paliy

Jiahao Pang

Sharathchandra Pankanti

George Papakostas

Vincent Paquit

Felipe Parages

Dongsun Park

Hyun Wook Park

Jong-Seung Park

Vorapoj Patanavijit

Jigisha Patel

Kedar Patwardhan

Marius Pedersen

Adithya Pediredla

Tao Peng

Yan-Tsung Peng

Federico Perazzi
Hayde Peregrina-Barreto

Hector Perez-Meana

Matthieu Perreira Da Silva

Said Pertuz

Renaud Peteri

Vladimir Petrovic

Tuan Pham

Jean-Charles Pinoli

Sankaranaryanan Piramanayagam

Hamed Pirsiavash

Lai-Man Po

Chiranjeevi Pojala

Stephen Pollard

C.J. Prabhakar

Ganesh Prasad

Jose Manuel Prats-Montalban

R. Pregitha

Aleka Psarrou

Raymond Ptucha

William Puech

Shrinivas Pundlik

Fei Qi

Jiang Qian

Jianzhao Qin

Minna Qiu

Regunathan Radhakrishnan

Venkatesh Babu Radhakrishnan

Christoph Raeth

Raghu Raj

Ajit Rajwade

Nicola Ranieri

Rene Rasmussen

Bradley Ratliff

Ajita Rattani

Ann Raynal

Carlo S. Regazzoni

Ulrich Reiter

Audrey Repetti

Gael Rigaud

Yong Ro

Carlos Rodriguez Pardo

Medhi Roopaei

Dalton Rosario

Christophe Rosenberger

Edward Rosten

Aditi Roy

Olivier Rukundo

Francesco Rundo

Marcal Rusinol

Abdelouahed Sabri

Firooz Sadjadi

Amit Sahu

Mohd Fadzli Mohd Salleh

Abbas Samani

Gustavo Sanchez

Hermilo Sanchez-Cruz

Raul Sanchez-Yanez

Bulent Sankur

José Santamaría

M.Saquib Sarfraz

M. Iqbal Saripan

Kaushik Sarkar

Hisao Sasai

Suchitra Sathyanarayana

Andreas Savakis

Firas Sawaf

Ediz Saykol

Jacob Scharcanski

Raimondo Schettini

Roland Schmitz 
Stuart Schweid

Ayan Seal

Gamal Seedahmed

Guna Seetharaman

Hojjat Seyed Mousavi

Ahamed Shafeeq

Soheil Shafiee

Mohammed Shahid

Shiguang Shan

P. Shanmugavadivu

Ling Shao

Mark Shaw

Hui-Liang Shen

Liquan Shen

Bin Sheng

Boxin Shi

Lilong Shi

Zhenwei Shi

Huang-Chia Shih

Ilan Shimshoni

Xianbiao Shu

Pushpa Mala Siddaraju

Khalid Siddiqui

Nils Siebel

Piyarat Silapasuphakornwong

Raimo Silvennoinen

Donggyu Sim

Harsh Singh

Marten Sjostrom

Irwin Sobel

Raffaele Solimene

Byung Cheol Song

Mingli Song

Humberto Sossa-Azuela

Thambipillai Srikanthan

Umamahesh Srinivas

Rajeev Srivastava

Nicolas Staelens

Roman Starosolski

Ove Steinvall

Ioannis M. Stephanakis

Vitomir Struc

Peter Stubler

Shahrel Azmin Suandi

Navneeth Subramanian

Vildana Sulic

Huifang Sun

Qiang Sun

Ting Sun

Xiaoshuai Sun

Zhenan Sun

Kyung Hyun Sung

Jayalakshmi Surendran

J. Edward Swan

Pinyo Taeprasartsit

Yu-Wing Tai

Kazunori Takahashi

Wing-Shan Tam

Samir Tamer

Ashish Tamhane

Songxin Tan

Masayuki Tanaka

He Tang

Jinshan Tang

Jean-Philippe Tarel

Ziya Telatar

Alptekin Temizel

Shyh Wei Teng

Andrew Teoh

Vanessa Testoni
Jukka Teuhola

Kalaivani Thangamani

Christos Theoharatos

K. K. Thyagharajan

Yonghong Tian

Yann Traonmilin

Hanene Trichili

Frederic Truchetet

Chih-Fong Tsai

Chien-Cheng Tseng

Efstratios Tsougenis

E. Turkel

Mohammad Shorif Uddin

Matthieu Urvoy

Hamid Reza Vaezi Joze

Preethi Vaidyanathan

Marc Van Droogenbroeck

Rahul Vanam

Sebastiaan Vanleuven

Michael Vannier

Sreenath Rao Vantaram

Damian Vargas Vazquez

Natalia Vassilieva

Mayank Vatsa

Javier Vazquez-Corral

Harini Veeraraghavan

Ragav Venkatesan

Steven Verrall

Claus Vielhauer

Dhanraj Vishwanath

Domenico Vitulano

Raluca Vlad

Benoit Vozel

Pradeep Vukkadala

Shu-Yen Wan

Cailing Wang

Chang-shuai Wang

Feng Wang

Han Wang

Hong-qiang Wang

Jian Wang

Jianhui Wang

Jing Wang

Jinwei Wang

Jun Wang

Junle Wang

Liang Wang

Lipo Wang

Meng Wang

Qiaosong Wang

Qunming Wang

Ruiping Wang

Wenmin Wang

Xu Wang

Yanfang Wang

Yu Wang

Z. Jane Wang

Zhifei Wang

Zhuozheng Wang

Nilanthi Warnasooriya

Steve E. Watkins

Simon Watt

Krzysztof Wegner

Geng Wei

Shiuh-Ku Weng

Gordon Wetzstein

Williem Williem

Stefan Winkler

Marcin Witkowski

Chee Sun Won
Tak-Shing Wong

Safwan Wshah

Ja-Ling Wu

Peng Wu

Qisong Wu

Yang Wu

Yue Wu

Zhenyu Wu

Ziyan Wu

Chunlei Xia

Chao Xiao

Feng Xiao

Han Xiao

Jin-Sheng Xiao

Liang Xiao

Song Xiao

Xiao Xiao

Kai Xie

Ning Xie

Rong Xie

Xiaohua Xie

Xudong Xie

Jin Xin

Sun Xin

Edmund Xing

Weihua Xiong

Chi Xu

Dongjiang $\mathrm{Xu}$

Li Xu

Qiong Xu

Ran Xu

Jingteng Xue

Toshihiko Yamasaki

Hao Yan

Ming Yan

Yanjun Yan

Bin Yang

Cong Yang

Dan Yang

Dong Yang

Jeehong Yang

Jie Yang

Shiyuan Yang

Tao Yang

Xuefeng Yang

Yi Yang

Guobiao Yao

Ming Yao

Feng $\mathrm{Ye}$

Seokwon Yeom

Changhoon Yim

August Yitzhak

Juha Ylioinas

JaeDoug Yoo

Soweon Yoon

YeoSun Yoon

Junyong You

Hongchuan Yu

Jin-Gang $Y u$

Shiqi $Y u$

Wurong Yu

Xuelian Yu

Jie Yuan

Xiaohui Yuan

Karim Zarei Zefreh

Guangtao Zhai

Xiaojun Zhai

Kun Zhan

Chi Zhang

Fan Zhang 
Hao Zhang

Jianming Zhang

Jing Zhang

Jing Zhang

Jun Zhang

Junping Zhang

Kaihua Zhang

Lei Zhang

Lin Zhang

Shengping Zhang

Shu Zhang
Weiwei Zhang

Xiangrong Zhang

Xianquan Zhang

Yi Zhang

Yudong Zhang

Zhijun Zhang

Zonghua Zhang

Yao Zhao

Sheng Zheng

Dexing Zhong

Guoyun Zhong
Jingbo Zhou

Jun Zhou

Xiran Zhou

Yahan Zhou

Jie Zhu

Yingying Zhu

Barbara Zitova

Vladimir Zlokolica

Peter Zolliker

Qin Zou 